Review

\title{
Spectroscopic Methods for Analysis of Nano Drug Distribution System
}

\author{
Hesham Salem ${ }^{1}$, Fatma Abo Elsoud ${ }^{1}$, Ahmed Magdy ${ }^{2}$, Dina Heshmat ${ }^{2}$, Ahmed Abdelkareem \\ Soliman ${ }^{2}$, Kerolos Wissa ${ }^{2}$, Kareem Hassan², Rania Abdelrady ${ }^{2}$ \\ ${ }^{1}$ Pharmaceutical Chemistry Departmenta, Faculty of Pharmacy, Deraya University, New Minia, Egypt. \\ ${ }^{2}$ Faculty of Pharmacy, Deraya University, New Minia, Egypt.
}

—Corresponding author. E-mail: fahmed_ch@yahoo.com, fatma.aboelsoud@deraya.edu.eg

Received: Jan. 19, 2020; Accepted: May 14, 2020; Published: Jun. 24, 2020

Citation: Hesham Salem, Fatma Abo Elsoud, Ahmed Magdy, Dina Heshmat, Ahmed Abdelkareem Soliman, Kerolos Wissa, Kareem Hassan, and Rania Abdelrady, Spectroscopic Methods for Analysis of Nano Drug Distribution System. Nano Biomed. Eng., 2020, I2(2): I67- 177. DOI: 10.5101/nbe.v12i2.p167-177.

\begin{abstract}
The size of the objects that are constituted within an alleged nano metric measurement of 1 to $100 \mathrm{~nm}$ is generally the main feature of nanoscience and nanotechnology. The system of unharness may be an essential method of acknowledging contained drug delivery systems in the formation of nano drug. Nanoparticles may surpass the crucial troubles of traditional little molecules / biomacromolecules, such as protein, ribonucleic acid, and DNA, utilized in several diseases through permitting objective delivery and surpassing biological obstacles. Many spectroscopical analytical techniques have been applied to define the free drug component from the nano drug formation, in various substantial cases, during various duration. One of them is analytical chemistry concerning the development of new techniques to develop old ones and supply the requirements of chemical information constrained by modern issues. Analytical chemistry is greatly affected by the development of nanoscience and nanotechnology. The aim of this review is to present a comparison of different spectroscopic analytical techniques which are presently applied to various systems of nano drug delivery to present elaborate and helpful data for other researchers.
\end{abstract}

Keywords: Spectroscopic analytical techniques; Nano drug distribution systems; Nano transporters; Drug release; Biological fluids; Pharmaceutical preparations

\section{Introduction}

The analytical methods mainly use nanotechnological techniques to employ their outstanding features of nanoparticles which are very effective in introducing entrenched analytical ways or developing old ones to seek new analytes or matrices. These nanoparticles help to develop the studied analytical system through improving characteristics of portability, rapidity, miniaturizability, and selectivity [1].

Presently, the formulations of nano drug are preferable due to their enhanced stability, bioavailability, and solubility, and their ability to decrease toxicity and to be directed to certain parts of the body. Due to their superior features, the formulations of nano drug are preferrable in many uses such as cancer therapies, imaging agents, vaccines nutraceuticals, and cosmetics [2].

The systems of nano drug distribution are of two different types: lipid or polymer-based according to the physicochemical features, the pharmacokinetics and the bioavailability of the drug. That can differ in 
these of two types of delivery systems. While the first type of nano drugs is pointed to lipid nanoparticles, now a respectable number of lipidic and polymeric are targeted nano drugs at markets and in clinical trials [3].

Nanomedicine is an evolving area of study which investigates the information utilization and methods of nanoscience in medical biology, malady interference, and treatment. Nanomedicine involves employing nanodimensional articles such as nanorobots, nanosensors in diagnosis, delivery, sensory objectives, and motivating substances in living cells (Fig. 1).

Fig. 2 contains summarized samples of polysaccharides applied in nanomedicine collected from various sources. The biopolymers success in nanomedicine and drug distribution is because of their versatility and other specific characteristics as they may result from soft gels, flexible fibers and hard shapes. Accordingly, they may be porous or non-porous. As well as they possess huge resemblance regarding the content of the extracellular matrix, which can avert immunological responses [5, 6].
Fig. 3 displays certain examples of biological compounds gained from higher plants and the application of these compounds in nanomedicine. Pharmaceutical productions seek the use of new technologies for developing and designing modern drugs and enhancing old ones [7, 8]. Accordingly, a design of new formulations in various fields; such as nanopharmaceutics (leading the drug to the location of action), nanodiagnostic (appearance and diagnosis), nanobiomaterials (medical implants), and nanotheranostics (the diagnosis combination and diseases treatment); has resulted from the development of nanotechnology [7, 9, 10].

Several analytical techniques are widely used for analyzing biological orders and preparing drugs. According to their facility of discovering little amounts in samples on semi, micro, and trace levels. They are also preferrable regarding nano drug formulations analysis.

The aim of the development of new drug formulations is improving bioavailability and

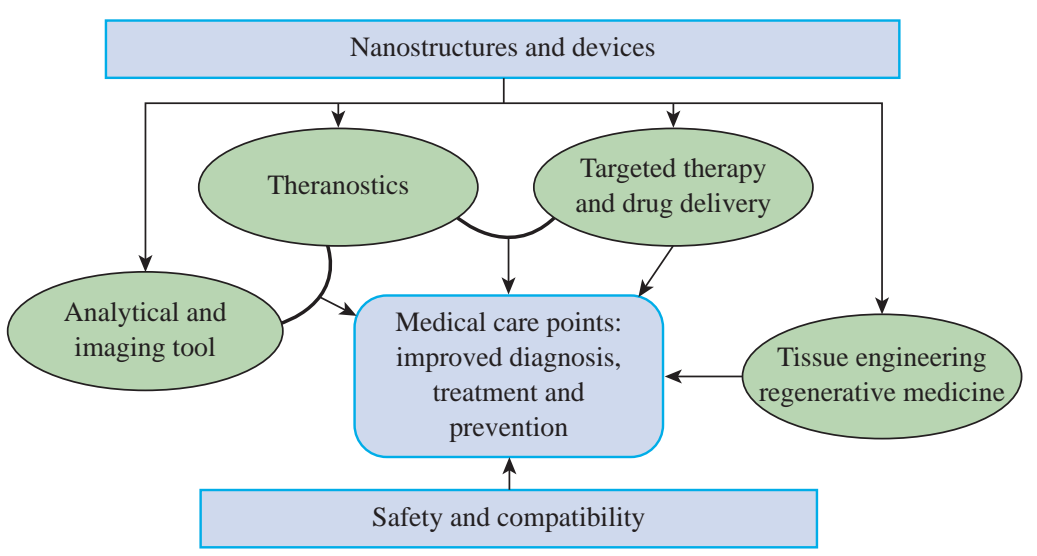

Fig. 1 Application and goals of nanomedicine in different sphere of biomedical research.

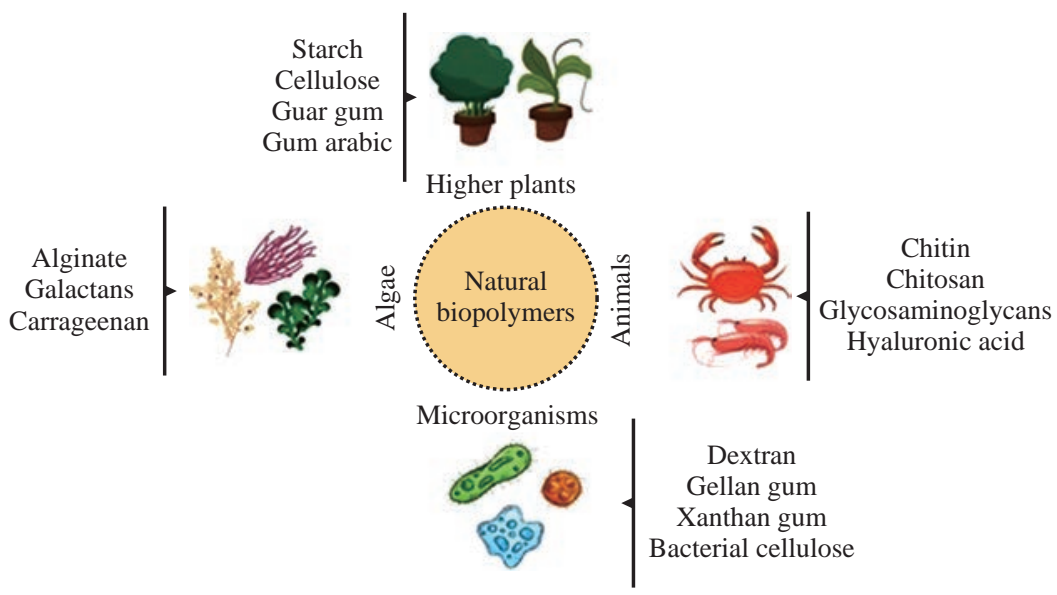

Fig. 2 Different sources of natural biopolymers to be used in nano medicine applications. 


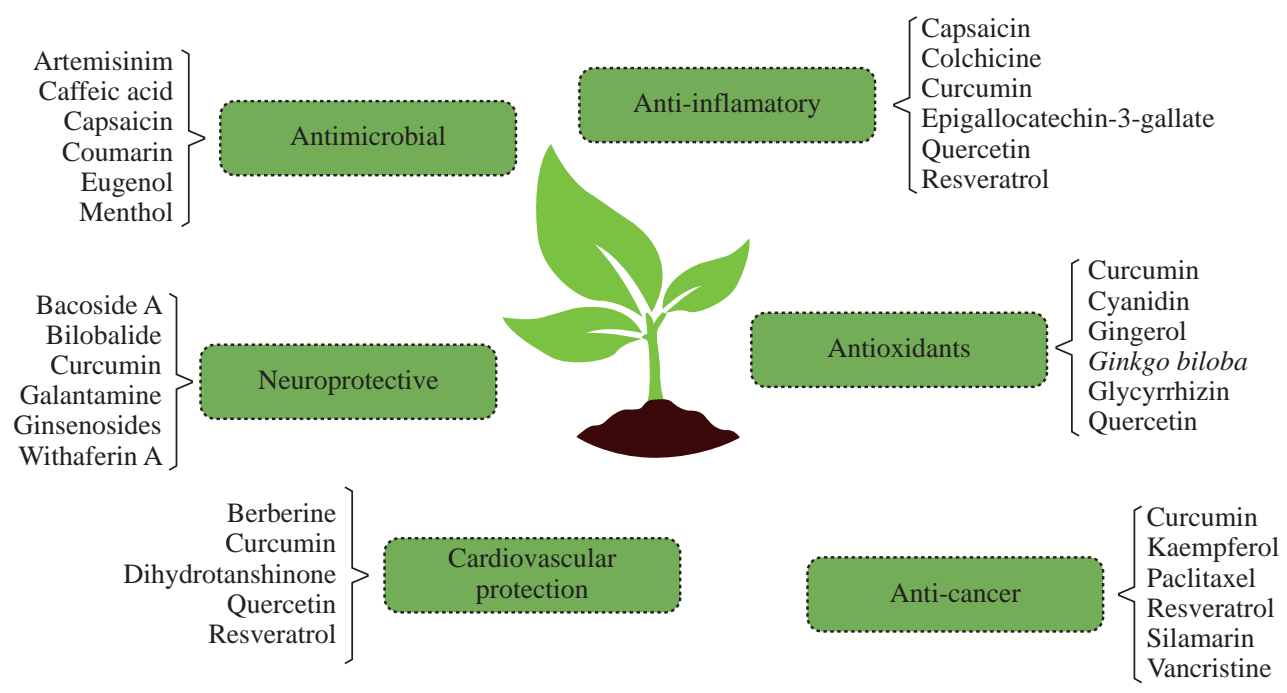

Fig. 3 Examples of natural compounds extracted from higher plants used in nanomedicine aiming different approaches. Some of these extracts are already being marketed, others are in clinical trials and others are being extensively studied by the scientific community.

decreasing the side effects. For assessing the therapeutic chance of preparing drug, analytical procedures are essential. The quantity of drug in urine, plasma, serum or in previous biological fluid or tissue supplies information of free therapeutic dose. Moreover, our aim of the review writing is to present several analytical methods for directing researchers to the consequences of nano preparations, and helping develop modern analytical techniques for the systems of nano drug.

Analytical nanosystems are described as implements or tools which possess a nanometric volume and are contained through the physico-chemical principles of nanoscience. Beside the nanometric volume, the exceptionality of nanomatter is used too. This resembles a great status because the micro components will be required to link between the nano-world and the macro-world [11].

\section{Spectroscopic Analytical Methods}

Numerous spectrophotometric methods were reported for purpose and analysis of several drugs by nanoscience and nanotechnology through their pure stage or their pharmaceutical formation. Several analytical techniques have been clarified for determination of certain drugs by nanoscience and nanotechnology.

\section{Ultraviolet-visible spectrophotometric methods}

Several ultraviolet-visible spectrophotometric methods were published for analysis of certain drugs after nanoparticulations either in bulk powders, pharmaceutical dosage forms and biological fluids [1240] as depicted in Table 1.

\section{Spectrofluorimetric methods}

Several spectrofluorimetric methods were published for analysis of certain drugs after nanoparticulations either in bulk powders, pharmaceutical dosage forms and biological fluids [41-50].

Teicoplanin (TEIC) is a glycopeptide antimicrobial medicine which is used to organize some bacterial infectious diseases instigated through gram-positive bacteria such as methicillin-resistant Staphylococcus aureus and Enterococcus faecalis. Original, actual plain, rapid and economical spectrofluorimetric methods were developed for the ultratrace determination of TEIC in the human plasma and the pharmaceutical vessels. The investigated methods based on defining the fluorescence of TEIC in methanol and strengthening its fluorescence by ten folds using silver nanoparticles (AgNPs) without any solvent extraction. The achieved techniques were applied to control TEIC in the plasma of humans with huge retrieval rate. Moreover, the suggested techniques were practical to examine the constancy of TEIC after the detection of different dissolution stress cases and kinetic dissolution [41].

Carbinoxamine antidepressant (CBX), that is, a communal component in the treatment preparations of cold and cough, is resolute by a responsive, humble and proper spectrofluorimetric technique regarding 
Table 1 Ultraviolet-visible spectrophotometric methods

\begin{tabular}{|c|c|c|c|}
\hline Analyte & Principle & $\lambda_{\max }(\mathrm{nm})$ & Ref. \\
\hline Crystal violet & $\begin{array}{l}\text { A surface enhanced Raman scattering substrate consisted of calcium alginate sponge incorporating gold nanoparticles. The } \\
\text { dye absorbed on the sponge can be detected. The consequences show that the lowest detectable concentrations. }\end{array}$ & 340 & [12] \\
\hline Bisoprolol and doxazosin & $\begin{array}{l}\text { Spectrophotometric method was progressed for determination of bisoprolol hemifumarate, buspirone } \mathrm{HCl} \text { and doxazosic } \\
\text { mesylate regarding pure shape and pharmaceutical expressions. This technique depended upon the aggregation of synthetic } \\
\text { gold nanoparticles. The technique was applied successfully to control the studied drugs in minor concentrations regarding } \\
\text { pure shape and pharmaceutical dosage forms. }\end{array}$ & $616-670$ & [13] \\
\hline
\end{tabular}

$\beta$-Lactams $\quad$ Resolve of cefotaxime, cefoperazone, ceftazidime and cefadroxil by reduction of $\mathrm{Ag}^{+}$ions to silver nanoparticles with the attendance of polyvinyl pyrooolidone as a stabilizing agent causing very strong surface plasmon echo.

Determination of two flavonoids within the separated portions obtained of Persicaria salicifolia, authentic and pharmaceutical dosage forms, the method depend on decreasing $\mathrm{Ag}^{+}$cations to silver nanoparticles (Ag-NPs) through the result of these Diosmin and rutin $\quad$ flavonoids in the presence of polyvinylpyrrolidone. The haemostatic action of the complete methanol extract, isolated
fractions, authentic and dosage forms were examined due to their usual and silver nanoparticulated shapes employing the time of tail bleeding and the amount of bleeding assays in rats.

Tacrine was formulated and loaded on poly(lactide-co-glycolide) nanoparticles in handling the alzheimer,s disease and

Tacrine $\quad$ were provided using modified nano precipitation technique. Pharmacodynamics studies of nanoparticles were estimated for brain and memory improvement in scopolamine motivated amnesic mice using morris water test and inhibitory step down avoidance.

Letrozole was determined by developing a magnetic nanoparticles combined ten generation oxime dendrimer concluded by polyethylene glycol as a drug extractant. The PEGylated dendrimer-based $\mathrm{MnPs} / \mathrm{SiO}_{2}$ were distinguished by Fourier convert infrared spectroscopy, filed release scanning electron microscopy, thermogravimetriv analysis and vibration sample magnetometer.

Letrozole

Silver nanoparticles are functional as topical coiled materials, accumulated precipitation of silver in liver, spleen and other Infected wounds $\quad$ principle organs can drive to organ injury and dysfunction. Treating mice using MRSA wound infection proved that the three kinds of AgNPs adequately evaluated MRSA wound infection and increased wound treating.

Anthelmintic drugs Application of silver nanoparticles as a colorimetric investigation for determination of three anthelmintic benzimidazole drugs. The method was founded on the AgNPs combination encouraged by their interaction through the cited drugs.

Determination of antimicrobial belongings of silver nanoparticles depended mainly on the surface plasmon echo groups.

Antimicrobial drugs $\quad$ Effects regarding several processing variables on the size and shape of AgNPs were mentioned using UV-visible spectrophotometer founded on their SPR bands.

Bacterial infections are an increasing problem worldwide. Washing hands play an important role in preventing bacterial

Antibacterial drugs infections, but other techniques as using nanoparticles-coated surfaces are required too. The antimicrobial features of the nanoparticle surfaces were measured with the touch test way against Escherichia coli and staphylococcus aureus.

Contemporary researches started to introduce structure and compositional studies as well as studies aimed at grasping the method of action about the studied material after nanoparticulation using the nanoparticles of silver and its ability as a drug bioenhancer.

Bhasma

Request of surface Plasmon echo of change state citrate capped silver nanoparticles to distinctively resolve many

Fluroroquinolones fluoroquinolone drugs.

Silver nanoparticles have been attentive on potential requests in cancer diagnosis and therapy. Synthesis and analysis and

Cancer sample analysis of AgNPs and methods for their classification were studied as anticancer agents.

Ethionamide

The main aim of contemporary education is to evolve a plain, particular economic UV-spectrophotometric technique to estimate Ethionamide in majority and nano marketed formulations.

In this work, the study of sedimentation velocity information of the characterized nano carrier drug delivery systems applied in nanomedicine. Nano carrier volume distribution and ratio of the free with the nanoparticle-encapsulated drug in a commercially available liposomal doxorubicin formulation are specified.

Due to its fast enzymatic dissolution within the gastrointestinal tract, insulin cannot be orally taken. Nanoparticulate

Insulin
insulin to degrade and facilitate insulin absorption via a transcellular or paracellular way.
In this education the strategy supported the flexibility of the cited drugs to scale back Ag+ions to silver nanoparticles (Ag-NPs)

Insulin
insulin to degrade and facilitate insulin absorption via a transcellular or paracellular way.
In this education the strategy supported the flexibility of the cited drugs to scale back Ag+ions to silver nanoparticles (Ag-NPs)

Insulin
insulin to degrade and facilitate insulin absorption via a transcellular or paracellular way.
In this education the strategy supported the flexibility of the cited drugs to scale back Ag+ions to silver nanoparticles (Ag-NPs)

Fenoterol and salbutamol with polyvinyl pyrrolidone (PVP) as a stabilizing factor making exceedingly acute surface plasmon echo peak of Ag-NPs. 417-425 Absorbing the Ag-NPs of the plasmon permits for measuring spectrophotometric discovery of the cited drugs.

Thiamine Determination of thiamine in solution by UV-Visible spectrophotometry: The result of interactions with gold nanoparticles.

The infusion of drug inside the skin reduces skin inflammation. To recognize the objective, the drug was formed in nanoparticles with chitosan resembling a polymer and the drug laden nanoparticles were inserted into gel.

Hydrazine Determination of hydrazine supported the tryptophane capped gold nanoparticles.

Fexofenadine Resolve of fexofenadine expending silver nanoparticles by spectrophotometric technique.

Nebivolol Discovery of nebivolol drug founded on as-grown un-doped silver oxide nanoparticles arranged with a wet- chemical way.

Antimicrobial Gold nanoparticles single physicochemical properties such as large external area to mass ratio and functionalizable structure. These properties can be practical to facilitate the administration of antimicrobial therapeutics.

Glutathione and cysteine Spectrophotometric determination of glutathione and cysteine founded on aggregation of mixture gold nanoparticles.

Drug carrier

UV-visible spectrophotometry to describe submicroscopic drug carriers such as nanoparticles and lipid vesicles was investigated. The framework of Rayleigh-Gans-Debye approximation, the preparations polydispersity and lamellarity and 400-600 monitored vesicle volume reforms was first used to examine it.

Hair drugs

The storage behavior as well as the penetration of dye-containing nanoparticles within the hair follicles were examined. The results were similar to the conclusions gained from a similar quantity of dye in the non-particle shape.

The determination of this education was to advance and describe a rigid lipid nanoparticle (SLN) method covering an anionic polymer to distribute chemosensitizers and cationic antineoplastic agents. Ionic complexation was used to strengthen the Verapamil polymer to distribute chemosensitizers and cationic antineoplastic agents. Ionic complexation was used to strengthen the
loading of the extremely water-soluble drugs. The influence of anionic compounds and polymers on drug division and loading using SLNs was examined. Dextran sulfate (DS) was the most appropriate among these deliberate . 
its pure type, pharmaceutical preparations and spiked plasma of humans. This way is based on the activity of the native fluorescence intensity of CBX in alcohol at a wavelength of 273/308 nm. Moreover, it is also used to investigate the stability of CBX in stress cases target-hunting during the International Conference of Harmonization (ICH). Up to now, there is no fluorescence-based method stated for CBX, even with the benefits of that method. This education tends to investigate the primary time and the CBX fluorescence attitude to propose new spectrofluorimetric technique that presents sensitivity and simplicity in antecedently printed reports. The projected technique displays a 0.66 $\mathrm{ng} \mathrm{mL} L^{-1}$ limit of detection and a $1.99 \mathrm{ng} \mathrm{mL}^{-1}$ limit of quantitation, that allows its application within the determination of CBX in the spiked plasma samples of humans for being quick and economical instrument in vitro examination with accepted results. The technique was proved in terms of robustness, precision, accuracy, andlinearity. The results indicated that there is a little distinction in accuracy and precision regarding the spectrophotometric comparison technique [42].

Polymertic magnetic ionic liquid-coated nanoparticles are classified as being adsorbents to the magnetic solid-phase extraction of slfuzosin, doxazosin, terazosin and prazosin, out of pharmaceutical preparations, plasma samples, and urine samples. The 4 kinds of drugs are detected through using fluorescence spectrophotometer at 350-500 $\mathrm{nm}$ [43].

Palashuddin et al. stated the results of the attendance of carbon nanoparticles (CNPs) inside several carbohydrate created food caramelssuch as sugar caramel, bread, jaggery, biscuits and corn flakes, in which their formation includes heating of the beginning substance. The CNPs were amorphous in nature; the particles were spheral with the sizes of 4-30 nm, relying on the extracting origin. The findings additionally revealed that particles with greater temperature are smaller than those shaped at less temperature. All the proposed samples achieved excitation tunable photoluminescence of quantum yield (QY) for CNPs: 1.2, for bread; 0.55 for jaggery; and $0.63 \%$ for sugar caramels. The current detection proposes the prospective utility of CNPs regarding different biological uses, because regular food items are used as sources of extraction. Most of these items $\mathrm{t}$ are used by humans centuries ago, accordingly, they will be regarded as secure [44].

Curcumin is recognized for playing neuroprotective role, acquiring antioxidant, as well as possessing inflammatory activities.

Rotenone, which is a flavonoid encouraged neurotoxicity within the dopaminergic cells, is greatly examined in Parkinson's disease investigation. Curcumin stuffed with lactoferrin nano particles organized through sol oil chemistry were applied for preserving dopaminergic cell line from rotenone encouraged neurotoxicity. These particles were 43$60 \mathrm{~nm}$ diameter size and about $100 \mathrm{~nm}$ hydrodynamic size as respectively evaluated by transmission electron microscopy, atomic force microscopy, and dynamic light scattering analysis. The efficiency of encapsulation was $61.3 \% \pm 2.4 \%$. Confocal imaging assured the cellular acceptance of curcumin with these nano particles. Spectrofluorimetric analysis of the curcumin stuffed with lactoferrin nanoparticles appeared higher intracellular drug absorbance, assisted retention, and better neuroprotection than their dissolved counterparts. Neuroprotective enterprise was recognized by viability tests and by evaluating Ross levels. Moreover, rotenone producing Pd like the one characteristics were recognized by reducing tyrosine hydroxylase expression and increasing a- syncline one. Taken together with curcumin loaded lactoferrin nanoparticles can be a favorable drug distribution technique against neurotoxicity in dopaminergic neurons [45].

Zeid et al. introduced a new effective fluorimetric way for the check of fexofenadine hydrochloride by silver nanoparticles as a probe of fluorescence. Adding a drug to the silver nanoparticles sol. resulted in great cooling of the emission band of silver nanoparticles, mostly caused by the complexation of the drug to the silver nanoparticles [46].

Magnetic nanoparticles are standard in medical specialty uses mainly for their use in echo imaging, magnetic physiological condition and targeted drug delivery. Comprehending the interactions of proteinnanoparticle within the stream of the blood has an a very important role because of their possible danger, specifically for immunogenicity. The interaction of nanoparticle-protein and also the formation of protein corona were investigated by chemical and biophysical approaches: supported dynamic lightweight scattering, gel dielectrolysis, circular dichroism spectrographic analysis, and differential scanning fluorimetry [47].

Nano-aggregates of N-acetyl-N-carboxymethyl chitosan $(\mathrm{NCac})$ are examined at $0.5 \mathrm{mg} \mathrm{mL}^{-1}$ victimization nucleus fluorimetry analysis in $0.1 \mathrm{~mol}$ 
$\mathrm{L}^{-1}$ phosphate buffer (pH 7.4). Dynamic light scattering and atomic force microscopy were used to determine the dimensions and morphology of the masses. The stabilization of the particles for $20 \mathrm{~h}$ in buffer was defined. Camptothecin was among the particles with different ways and the rate constant for drug release $(\mathrm{k})$ was also defined. Strong interactions between the drug and the hydrophobic core of the compound micelles were recognized by lower $\mathrm{k}$ values [48].

Synthesis of poly (sulfobetain methacrylate) (p (SBMA)) hydrogels of micro dimensions using inverse suspension polymerization of a zwitterionic monomer 2- dimethyl (3-sulfopropyl) ammonium hydroxide (SBMA) was studied. The provided microgels are expended for being microre-(methacryloyloxy) ethyl agents for the synthesis of nickel (Ni) nanoparticles through in situ reduction of $\mathrm{Ni}$ (II) loaded within microgels out of the aqueous channel. The provided microgels as well as the microgel metal nanoparticle compounds were identified using transmission electron microscopy (TEM), scanning electron microscopy (SEM), and Fourier transformation infrared (FTIR) spectroscopy. Thermal features of microgels were examined using Thermal Gravimetric Analysis (TGA). The quantity of Ni nanoparticles produced in the microgels was recognized by Atomic Absorption Spectroscopy (AAS) after liquefying the Ni nanoparticles to shape Ni (II) ions through their treatment with concentrated hydrochloric acid $(5 \mathrm{M} \mathrm{HCl})$. The provided compounds were applied as catalysts to reduce nitro aromatic compounds such as 4-nitrophenol (4-NP), 2-nitrophenol (2-NP) and 4-nitroaniline (4-NA) and as a result, excellent catalytic performances were noticed. The belongings of temperature and the quantity of catalyst were also assessed. A weak activation energy, compared to the literature, was measured as $35.64 \mathrm{~kJ} / \mathrm{mol}$, and a very high kapp value as a function of temperature was evaluated to reduce 4-NP catalyzed through $\mathrm{p}$ (SBMA)-Ni composite catalyst order [49].

Solid-phase removal is one of the most valuable and useful methods for preparing, purifying, preconcentrating samples, and determining of heavy metals at levels of trace. Regarding this review, to determinate trace of nickel, cadmium, lead, and copper in water as well as in seafood samples, functionalized MCM-48 nanoporous silica and 1-(2-pyridylazo)2-naphthol was practically used. The exploratory circumstances such as sample and eluent flow rate, $\mathrm{pH}$, concentration and volume of the eluent, type, effect of coexisting ions, and advance volume are enhanced for effective solid-phase extraction of trace heavy metals in various water and samples of seafood. The solutions content including the stated heavy metals was specified using flame atomic absorption spectrometry (FAAS), and the detection limits were $0.3,0.4,0.6$, and $0.9 \mathrm{ng} \mathrm{mL}^{-1}$ for cadmium, copper, nickel, and lead, respectively. Retrievals and precisions were $>98.0$ and $<4 \%$, respectively. The adsorption capacity of the developed nanoporous silica was $178 \mathrm{mg} \mathrm{g}^{-1}$ for cadmium, $110 \mathrm{mg} \mathrm{g}^{-1}$ for copper, $98 \mathrm{mg} \mathrm{g}^{-1}$ for nickel, and $210 \mathrm{mg} \mathrm{g}^{-1}$ for lead. The functionalized MCM-48 nanoporous silica with 1-(2-pyridylazo)-2-naphthol was identified by thermogravimetry analysis (TGA), differential thermal analysis (DTA), transmission electron microscopy (TEM), Fourier transform infrared spectrometry (FTIR), X-ray diffraction (XRD), elemental analysis (CHN) and $\mathrm{N}_{2}$ adsorption surface area measurement [50].

\section{Atomic absorption spectroscopy}

Several atomic absorption spectroscopic techniques were published for analysis of certain drugs after nanoparticulations either in bulk powders, pharmaceutical dosage forms and biological fluids [5153]. Buse et al. described the determination of metal at trace levels by slotted quartz tube flame atomic absorption spectrophotometry (SQT-FAAS) after preconcentration by the presence of stearic acid coated magnetic nanoparticle (SAC-MNPs) based sonication assisted dispersive solid phase extraction (SADSPE). SAC-MNPs were applied according to their informal separation characteristics by the request of external magnetic field. All extraction parameters were improved through response surface method founded exploratory design. The experimental information was estimated through the analysis of alteration. In the optimum cases, nearly thirty-one enhancing folds regarding the power of detection was gained over the traditional FAAS [51].

Jade et al. introduced the use of gold nanoparticles that, according to their small size, may be managed smoothly through intravenous injection. The clinical utility of nanoparticles can be determined through the capacity to enter cells. TEM recognized the size of gold nanoparticle. The analysis of intracellular approval regarding dissimilar sizes of gold nanoparticle, development periods, and concentrations were identified through the use of atomic absorption spectrometry (AAS). The findings revealed that 
pancreas cancer cells absorb 20 nm gold nanoparticles in comparison with the other sizes of gold nanoparticle. Effective buildup of gold nanoparticles into the case of pancreas cancer cells may be realized at longer development span of time and advanced concentration. Results of that education help in designing and optimizing the gold nanoparticle-based agents for therapeutic and diagnostic uses of X-ray drug delivery system [52].

Applying zincon-immobilized silica-coated magnetic $\mathrm{Fe}_{3} \mathrm{O}_{4}$ nanoparticles (Zincon-Si-MNPs) as solid-phase removal (SPE) medium was useful in the isolation and preconcentration of suggestion lead in water. Many parameters such as extraction time, $\mathrm{pH}$, sample size, concentration and size of eluent, and effect of co-existing ions are examined to build the optimum cases for the strength of lead in mixing with graphite furnace atomic absorption spectrometry (GFAAS). The limit of discovery (LOD) of the introduced technique for lead on the basis of an improvement factor of 200 was $10 \mathrm{ng} \mathrm{L}^{-1}$. The relative standard deviations (RSDs, $\mathrm{n}=5$ ) were $8.3 \%, 7.8 \%$ and $9.2 \%$, respectively, at 5, 0.5 and $0.05 \mathrm{ng} \mathrm{mL}^{-1}$ levels. This technique has been effeciently used in the analysis of trace lead in the samples of drinking and natural water and $84-104 \%$ was the range of the recoveries for the spiked samples [53].

\section{Infrared spectroscopy}

The magnetic $\mathrm{FeNi}_{3}, \mathrm{SiO}_{2}, \mathrm{TiO}_{2}$ nanoparticles are produced with co-precipitation and sol-gel techniques and applied as a photocatalyst object for the elimination of humic acid compounds (HA) within various experimental parameters. The synthesized catalyst is distiguished by analyzing TEM images; FE-SEM images; XRD patterns; FT-IR spectra; EDS patterns, andVSM techniques. The findings revealed the effective synthesis of $\mathrm{FeNi}_{3}, \mathrm{SiO}_{2}, \mathrm{TiO}_{2}$ nanoparticles of excellent catalytical features: powerful functional classes, perfect crystallization, strong magnetic power, and nanosized number of particle diameter. The dissolution stage was sensitive under the diversity of experimental cases [54].

The magnetic metal-organic framework $\mathrm{Fe}_{3} \mathrm{O}_{4}$. (Fe-(benzene-1,3,5-tricarboxylic acid) (MMOF) is formulated, identified and examined as being a magnetic sorbent towards the dispersive solid-phase extraction (DSPE) of many applied regulators of blood lipid (i.e., clofibric acid, bezafibrate, gemfibrozil, clofibrate and fenofibrate) regarding samples of water. Distinguishing the synthesized $\mathrm{Fe}_{3} \mathrm{O}_{4}$. FeBTC magnetic nanomaterial is made through Fourier transform infrared spectroscopy, scanning electron microscopy, thermogravimetric analysis, powder X-ray diffractometry and transmission electron microscopy. The magnetic nanocomposite was observed for its chemical stablility, its large surface area $\left(803.62 \mathrm{~m}^{2}\right.$ $\left.\mathrm{g}^{-1}\right)$, and its pore volume $\left(0.59 \mathrm{~cm}^{3} \mathrm{~g}^{-1}\right)$. The fibrates concentrations in various water samples are found by HPLC-UV-Vis and affirmed by UPLCMS/ MS. The parameters that affect the extraction adequacy of magnetic-DSPE are examined and modified [55].

Encapsulation of Clove Essential Oil (CEO) by Chitosan Nanoparticles (ChNPs) is achieved, through postponement ionic gelation method to modify the antifungal CEO effectiveness. The mass ratios of chitosan $(\mathrm{Ch})$ to tripolyphosphate (TPP), 1:1, for unloaded ChNPs and 1:1:1 for Ch to TPP to CEO, for CEO-loaded ChNPs (CEOChNPs), were chosen as optimum preparations depending on ultravioletvisible spectroscopy and dynamic light scattering. The existance of CEO in optimum CEO-ChNPs, was marked by Fourier transform infrared spectroscopy [56].

AFM-IR, a photothermal method, syndicates AFM and infrared (IR) spectroscopy to unmistakably classify the arrangement of a sample chemically by tens of nanometer spatial resolution. Until now, it is effectively applied in interaction process in many uses. Nevertheless, the interaction process is inappropriate for adhesive or soft samples such as polymeric nanoparticles (NPs) of less than $200 \mathrm{~nm}$, there has been great interest for biomedical uses. In this paper, we explain the theoretical foundation of the advanced drumming AFMIR method that may address new contests in photographing and chemical mapping. The innovative technique supplies achievement data on NP morphology and arrangement, also it uncovers the site of the drug and the structures of the core-shell. Untit now, the sites of NP mechanisms can only be theorized, tapping AFM-IR permits for exactly imaging the site of the NPs' shells and the integrated drug. The special the drug accumulation in the NPs' top layers is demonstrated howerer, its little concentration ( $<1 \mathrm{wt} \%)$. These studies help in the use of tapping AFM-IR as a useful method to rule the quality of NP formulations depended on component quantification and individual NP detection [57].

Magnetic oxide nanoparticles resemble a wonderful 
instrument for biological and medical research, working asmagnetic carriers, contrast agents, and core materials of theranostic systems. While the requests depend mainly on iron oxides, more complicated oxides such as perovskite manganites can deliver a better magnetic enactment. For measuring the danger of their possible practice, the toxicity of manganite nanoparticles was fully examined and associated with the potential system of Mn-Zn ferrite nanoparticles. There are two main methods for preparing magnetic nanoparticles: the molten salt synthesis and the traditional sol-gel route. While nanoparticles of ferrite resembled the comparative material, they were synthesized by a new process under hydrothermal conditions. Magnetic cores are covered with silica and many examples of manganite nanoparticles with various thicknesses of silica shell were made [58].

Magnetic chitosan nanoparticles and chitosan nanoparticles are considered to be useful systems of distribution for anti-Alzheimer drug tacrine. Examination was applied to clarify the effect of process parameters on the ordinary particle size of chitosan nanoparticles made by casual emulsification. The method was modified by means of experiments design (DOE) by using a 3-factor, 3-level Box-Behnken statistical project. This design is applied to attain the smallest size and the most appropriate nanoparticles morphology. The optimal method was used to create magnetic chitosan nanoparticles. The intended nanoparticles possess regular particle size ranging from 33.64 to $74.87 \mathrm{~nm}$, that are determined by field emission scanning electron microscopy (FE-SEM). Drug loading in the nanoparticles as drug distribution systems is made by the presented optimal technique and the suitable capacity of drug loading was displayed by ultraviolet spectrophotometry. Diffuse reflectance Fourier transform mid infrared spectroscopy (DRFTMIR) characterize chitosan and magnetic chitosan nanoparticles as drug delivery systems [59].

Piyasi et al. advanced self-assembled chitosan/ insulin nanoparticles to successfully oral distribute insulin. The main aim of our study is preparing chitosan/insulin nanoparticles through selfassembly technique, to describe them and estimate their effectiveness in a vivo diabetic model. The nanoparticles scope and morphology are examined using scanning electron microscopy (SEM), atomic force microscopy (AFM) anddynamic light scattering (DLS). The regular size of the particle ranges from 200 to $550 \mathrm{~nm}$ in spherical or subspherical form.
An average of $\sim 85 \%$ was in the nanoparticles of insulin encapsulation. In vitro issue education, the nanoparticles were effective in absorbing satisfying amount of insulin in replicated gastric case, whereas obvious quantity of insulin release was noticed in artificial intestinal case. The oral managements of chitosan/insulin nanoparticles were useful in decreasing the blood glucose level of alloxan-induced diabeticmice. Hence, self-assembled chitosan/insulin nanoparticles appear satisfying features as possible insulin carrier system in animal samples [60].

Nagavaram et al. introduced a formulation study to improve the penetration of drug into the skin and the reduction of the skin agitation. To attain this goal, the drug was formulated with nanoparticles using chitosan as a polymer and this drug loaded nanoparticles were integrated in gel. The equipped nanoparticles were distinguished by SEM, DSC, FTIR, and the size of the particle. The size of the particle for elevated nanoparticulate gel (NPG-4) was between 49 and $305 \mathrm{~nm}$. SEM pictures revealed that nanoparticles hardly had spherical shape and free blows. The NPG4 introduced $43.9 \%$ of encapsulation effectiveness and $18.9 \%$ of drug loading. At $24 \mathrm{~h}$ expiration, the in vitro drug relief was noticed as $90.1 \%$ in $\mathrm{pH} 7.4$ phosphate buffer saline (PBS). In vitro skin penetration studies NPG-4 appeared $2.1 \mathrm{mg} \mathrm{cm}-2$ of drug penetration, which is better than the promoted cream formulation (NIZRAL 2\%), which appeared $1.2 \mathrm{mg} / \mathrm{cm} 2$ only of drug penetration. The NPG-4 displayed no initial skin agitation when tested on the skin of rabbit [61].

Attention is paid to hybrid magnetic hydrogels through applying them within biomedical science through systems of controlled drug delivery. We advanced a technique to gain new hybrid hydrogels within magnetic nanoparticles (NPs) of $\mathrm{CoFe}_{2} \mathrm{O}_{3}$ and $\mathrm{Fe}_{3} \mathrm{O}_{4}$ as cross linker carboxy agents of methyl cellulose (CMC) or hyaluronic acid (HYAL) polymers. We verified those systems for the purpose of releasing controlled doxorubicin. Magnetic NPs have been functionalized with (3-aminopropyl) trimethoxy silane (APTMS) for presenting amino groups surfacially. Amino coating has been identified and counted through X-ray photoelectron spectroscopy and standard Fourier transforming infrared spectroscopy techniques, and by cyclic voltammetry, a new method that allows us to see the solution characteristics of the functionalized NPs [62].

The magnetic nanoparticles were planned to be used 
for biomedical aims within a great extent for many years. The progress of mechanisims which would deliver drug molecules selectively to the diseased place, without a synchoronous level rise within healthy tissues, is now one of the most active cancer research areas. Conjugate carboxymethyl starch (CMS)/SPIO nanoparticles have been organized through chemical reaction. Numerous parameters such as the drug/ polymer ratios within the range of 1:14 have been observed for optimizing formulation. Morphology and size delivery of nanoparticles and in vitro releasing outline through the medium of phosphate buffer ( $\mathrm{pH}$ 7.4) during 12 h have been examined then. Magnetic NPs which were prepared within this education have been spherical through a relatively mono- isolated distributing size. FT-IR spectrum of pure carboxymethyl starch (CMS) and CMS-SPIONs, respectively, in which the percentage of transmission has been plotted as a technique of wave number $\left(\mathrm{cm}^{-1}\right)$. Wide peak around $3411 \mathrm{~cm}^{-1}$ has been ascribed into the (OH-) spreading CMS vibrations. The 1597 and 1417 $\mathrm{cm}^{-1}$ peaks attribute with the (COO-) symmetrical and unsymmetrical spread of vibrations in a corrisponding way [63].

\section{Chemometry}

Spectroscopy is extensively used to identify pharmaceutical products or procedures, specifically because of its preferable features of being fast, economical, non-invasive/non-destructive and suitable off-line and on-line. Spectroscopic methods produce forms containing huge amount of information, that may be beneficially used by the application of multivariate mathematic and statistic (chemometric) methods. This research aims at providing a summarized view of the various chemometric techniques used in the context of spectroscopy-based pharmaceutical analysis, discussing both the free examination of the provided information and the ability of making predictive models for both quantitative (calibration) and qualitative (classification) responses [64]. Functionalized magnetic nanoparticles are employed in many biomedical uses, such as magnetic cell separation, drug delivery and magnetic echo picturing. Size and surface belongings of iron oxide nanoparticles are the two significant factors that can deeply influence the nanoparticle effectiveness and stability. In this paper, the chemometrics attitude was used to strengthen the coating process of iron oxide nanoparticles. To strengthen the nanoparticles size, the impact of 2 size experimental parameters was examined through means of multivariate analysis. The factors evaluated are chitosan-to-tripolyphosphate concentration ratio and chitosan molecular weight. The experiments were done due to face-centered cube central composite response surface target. A secondorder regression model was found to have both descriptive and predictive aptitudes. The technique was modified regarding the percent of $Z$ average diameter's increasing after covering as reaction. It can be said that experimental design gives a appropriate means of modifying and challenging the robustness of iron oxide nanoparticle coating technique [65].

\section{Conclusions}

Nanoparticles are mostly used in the development of certain drugs used for treatment of certain diseases resulting in fewer harmful side effects. Also, nanoparticle formulations had the availability to improve the bioavailability of certain biological systems for anti-cancer, anti-inflammatory, antihypertensive, hypoglycemic, antibacterial, antivirus, antipsychotic, nutraceuticals etc. As reported and mentioned in the survey of the literature, different spectroscopic techniques are usually wanted due to the sensitive and selective analysis of nano-formulations. Spectroscopic methods have been applied to identify the loaded quantity of a drug or the freed amounts. Regarding the literature survey, it is obviously known that ultraviolet-visible spectrophotometric technique is mostly preferred technique for assay of nano formulations.

Finally, it is clearly seen that spectroscopic techniques are selected techniques to evaluate the efficiency of nano formulations and can be applied easily and effectively in quality control labs with a low cost compared with other techniques like chromatographic techniques.

\section{Conflict of Interests}

The authors declare that no competing interest exists.

\section{References}

[1] P. Poole, F.J. Owens, Introduction to Nanotechnology. Wiley, 2003.

[2] R.H.C. Richard, W.R. Dennis, Nanotechnology in Medicine and the Biosciences, Developments in Nanotechnology, Vol. 3. Gordon \& Breach, 1996. 
[3] M.A. Theresa, R.C. Pieter, Liposomal drug delivery systems: From concept to clinical applications. Adv. Drug Deliv. Rev., 2013, 65(1): 36-48.

[4] K.P. Jayanta, D. Gitishree, F.F. Leonardo, et al., Nano based drug delivery systems: recent developments and future prospects. J. Nanobiotec., 2018, 16(71): 1-33.

[5] G.M. Bassas, S.P.M. Follonier, and M. Zinn, Natural polymers: a source of inspiration. In: Bioresorbable polymers for biomedical applications. Elsevier, 2017: 3164.

[6] M.J. Cardoso, R.R. Costa, and J.F. Mano, Marine origin polysaccharides in drug delivery systems. Mar Drugs, 2016, 14: 34

[7] B. Pelaz, C. Alexiou, R.A. Alvarez-Puebla, et al., Diverse applications of nanomedicine. Acs Nano., 2017, 11: 23132381.

[8] V. Grazu, M. Moros, and C. Sánchez-Espinel, Nanocarriers as nanomedicines: design concepts and recent advances. In. Frontiers of nanoscience, Vol. 4, Elsevier, 2012: 337-440.

[9] L.Y. Rizzo, B. Theek, G. Storm, et al., Recent progress in nanomedicine: therapeutic, diagnostic and theranostic applications. Curr Opin Biotechnol, 2013, 24: 1159-1166.

[10] T. Devasena, Diagnostic and therapeutic nanomaterials. In. Therapeutic and diagnostic nanomaterials. Springer, 2017: 1-13.

[11] M. Valcarcel, B.M. Simonet, S. Cardenas, Anal. Bioanal. Chem., 2008, 391: 1881-1887.

[12] F.U. Huiping, C. Jiaming, C. Lijuan, et al., Calcium aliginate sponge with embedded gold nanoparticles as a flexible SER substrate for direct analysis of pollutant dyes. Micrichimica Acta, 2019, 186: 64-70.

[13] M.A. Magda, E.A. Hisham, M.H. Mervat, et al., Aggregation of gold nanoparticles for spectrophotometric determination of bisoprolol hemifumarate buspirone $\mathrm{HCl}$ and doxazosin mesylate. Nano Biomed. Eng., 2019, 11(1): $1-10$.

[14] S. Hesham, S. Ebtihal, Determination of cefotaxime, cefoperazone, ceftazidime and cefadroxil using surface plasmon resonance band of silver nanoparticles. Braz. J. Pharm.Sci., 2018, 54(3): 1-9.

[15] S. Hesham, A.E. Atef, and M.A. Nevein, Utility of silver nanoparticles for the analysis of diosmin and rutin in Persicaria salicifolia extract, authentic and pharmaceutical dosage forms monitored with their haemostatic activity. Afr. J. Pharm. Pharmacol., 2018, 12(20): 248-262.

[16] V.J. Felix, S.K. Sathesh, Formulation, characterization and determination of anti-alzheimeric activity of tacrine loaded poly(lactide-glycolide) nanoparticles. Int. J. Pharm. Sci. Res., 2018, 9(12): 5111-5120.

[17] P.H. Shervin, Z.M. Ramin, A.P. Homayon, et al., Synthesis and characterization of pegylated dendrimers based on magnetic nanoparticles for letrozole extraction and determination in body fluids and pharmaceutical samples. Micrichimica Acta., 2018, 143: 190-197.

[18] P. Yinbo, S. Chenlu, Y. Chuanfeng, et al., Low molecular weight chitosan-coated silver nanoparticles are effective for the treatment of MRSA-infected wounds. Int. J. Nanomed., 2017, 12: 295-304.

[19] M.D. Sayed, R.H.A. Hassan, A.H. Ahmed, et al., Application of silver nano-particles for the spectrophotometric determination of three benzimidazole anthelmintic drugs in their pharmaceutical preparations. $J$. Appl. Pharm. Sci., 2017, 7(2): 76-82.

[20] G.M. Nichrous, S.S. Hulda, H. Askwar, et al., Antimicrobial dependence of silver nanoparticles on surface plasmon resonance bands against Esceherichia coli. Nan.Sci. Appl., 2017, 10: 1-9.

[21] G. Marianne, H. Janne, J.B. Kofi, et al., Antimicrobial characterization of silver nanoparticle-coated surfaces by touch test method. Nano. Sci. Appl., 2017, 10: 137-145.

[22] M. Sirisha, C. Vijay, and R.S. Bal, Physico-chemical analysis of herbally prepared silver nanoparticles and its potential as a drug bioenhancer. OpenNano, 2017: 19-27.

[23] M.D. Sayed, A.O. Mahmoud, A.H. Mohamed, et al., Application of surface plasmon resonance of citrate capped silver nanoparticles for the selective determination of some fluoroquinolone drugs. J. Appl. Pharm. Sci., 2017, 7(2): 16-24.

[24] F.Z.Xi, G.L. Zhi, S. Wei, et al., Silver nanoparticles:synthesis, characterization, properties, applications and therapeutic approaches. Int. J. Mole. Sci., 2016, 17: 1534-1551.

[25] K.D. Sujit, S. Saisivam, and D. Monalisha, Validated UVspectrophotometric method for the ethionamide estimation in bulk, tablet and nanoparticles. Int. J. Drug. Dev., 2017, 9(1): 20-23.

[26] M. Dora, I. Patrizia, C. Noelia, et al., Analytical ultracentrifugation for analysis of doxorubicin loaded liposomes. Int. J. Pharm., 2017, 523: 320-326.

[27] Milind S. A., Wen J.L., Shailaja S. P. Application of polymeric nanoparticles and micelles in insulin oral delivery. J. Food Drug Anal., 2015(23): 351-358.

[28] M.A. Magda, E.A. Hisham, M.H. Mervat, et al., Determination of etilefrine hydrochloride, fenoterol hydrobromide, salbutamol sulphate and estradiol valearate using surface plasmon resonance band of silver nanoparticles. Int. J. Phar. Sci., 2015, 7(5): 327-333.

[29] Z. Michael, Determination of thiamine in solution by UVVisible spectrophotometry: The effect of interactions with gold nanoparticles. Thesis submitted to the department of chemistry. Eastern Michigan University in partial fulfillment requirements for the degree of Master of Science in chemistry, 2014.

[30] B.V.N. Nagavarm, S.Y. Hemantk, S. Hemanth, et al., Formulation of polymeric nanoparticulate gel for topical delivery. Int. J. pol. Mat. Pol. Biomat., 2014, 63: 439-447.

[31] W. Gao, Y. Chen, and S. Xiao, Hydrogen-bonding recognition - induced colorimetric determination of hydrazine based on the tryptophan capped gold nanoparticles. J. Spec., 2013: 1-7.

[32] M.R. Rahnama, Determination of fexofenadine using silver nanoparticles by spectrophotometric method. Int. J. Chem. Tec. Res., 2013, 5(5): 2508-2512.

[33] M.M. Rahman, S.B. Khan, A.M. Asiri, et al., Detection of nebivolol drug based on as-grown un-doped silver oxide nanoparticles prepared by a wet-chemical method. Int. J. Eletro Chem. Sci., 2013, 8(2): 323-325.

[34] A. Azam, S. Mojtaba, H. Zahra, et al., Development of gentamicin-gold nanosphere for antimicrobial drug delivery to staphylocooal infected foci. Drug. Deliv., 2013, 20(1): 34-39.

[35] M.R.N. Hormozi, E. Seyedhosseini, and H. Robatjazi, Spectrophotometric determination of glutathione and cysteine based on aggregation of colloidal gold nanoparticles. Scientia Iran. F., 2012, 193: 958-963.

[36] X. Qin, D. Shi, and J. Gen, Determination of acetamipride by a colorimetric method based on the aggregation of gold nanoparticles. Microchim Acta., 2011, 173: 323-329.

[37] J. Tashkhourian, M.R.H. Nezhad, and J. Khodaveisi, Application of silver nanoparticles and principal component-artificial neural network models for simultaneous determination of levodopa and benserazide hydrochloride by a kinetic spectrophotometric method Spectrochim Acta Part A., 2011, 82(1): 25-30.

[38] M.A.E. Mustafa, C. Gregor, Turbidity spectroscopy for characterization of submicroscopic drug carriers, such as nanoparticles and lipid vesicles: size determination. Pharm. Res., 2011, 28: 2204-222.

[39] L. Juergen, R. Heike, T. Alexa, et al., Nanoparticles- An 
efficient carrier for drug delivery into the hair follicles. Eur. J. Pharm. Biopharm., 2007, 66: 159-164.

[40] L.W. Ho, B. Reina, M.R. Andrew, et al., Development of solid lipid nanoparticles containing ionically complexed chemotherapeutic drugs and chemosenesitizers. J. Pharm. Sci., 2004, 93: 1993-2008.

[41] F.B.A. Marwa, A.M. Mostafa, I.S. Baher, et al., Utility of surface plasmon resonance response of silver nanoparticles for assay of Teicoplanin in human plasma using spectrofluorimetric technique. Microchem. J., 2019, 146: $187-191$.

[42] A.A. Fatma, E.E. Nahed, E. Heba, et al., A fast spectrofluorimetric method for determination of carbinoxamine maleate in the nano-molar range. Application to pharmaceutical preparations, biological fluids and stability studies. Anal. Methods, 2018, 10: 3851-3858.

[43] G. Xiaozhen, W. Hao, G. Shiwen, et al., Highly sensitive fluorescence methods for the determination of afluzosin, doxazosin, terazosin and prazosin in pharmaceutical formulations, plasma and urine. Anal. Sci., 2016, 32: 763768.

[44] S. Palashuddin, J. Amit, P. Anumita, et al., Presence of amorphous carbon nanoparticles in food caramels. Sci. Rep., 383(2012): 1-5.

[45] V.S. Bollimpelli, P. Kumar, S. Kumari, et al., Neuroprotective effect of curcumin loaded lactoferrin nano particles against rotenone induced neurotoxicity. Neurochemistry International, 2016.

[46] A.A. Zeid, B. Nausheen, H. Sajjad, et al., Spectrofluorimetric determination of fexofenadine hydrochloride in pharmaceutical preparation using silver nanoparticles. Arab. J. Chem., 2010, 3: 351-355.

[47] M. Ulrike, B. Dominiqu, T. Delphine, et al., Maghemite nanoparticles stabilize protein corona formed with transferrin presenting different iron-saturation levels. Nanoscale, 2013: 1-9.

[48] M. Ajmal, S. Demirci, M. Siddiq, et al., Nurettin Sahiner. Betaine microgel preparation from 2-(methacryloyloxy) ethyl dimethyl (3-sulfopropyl) ammonium hydroxide and its use as catalyst system, Colloids and Surfaces A: Physicochem. Eng. Aspects, 2015, 486: 29-37

[49] J. Abolhasani, M. Behbahani, Application of 1-(2-pyridylazo)-2-naphthol- modified nanoporous silica as a technique in simultaneous trace monitoring and removal of toxic heavy metals in food and water samples, Environ Monit Assess, 2015, 187: 4176.

[50] P.B. Helen, V. Francine, R.S. Maria, et al., Formation, drug-release kinetics and solution-stability of N-acetylNcarboxymethyl chitosan nanoparticles as potential drug carriers. Int. J. Drug Del., 5 (2): 214-223.

[51] T.Z. Buse, F.E. Ahmet, S.C. Dotse, et al., Combination of stearic acid coated magnetic nanoparticle based sonication assisted dispersive solid phase extraction and slotted quartz tube-flame atomic absorption spectrophotometry for the accurate and sensitive determination of lead in red pepper samples and assessment of green profile. Food Chem, 2019: 1-19.

[52] D.T. Jade, M. Kazue, Y. Noritaka, et al., Size, concentration and incubation time dependence of gold nanoparticles uptake into pancrease cancer cells and its future application to X-ray drug delivery system. $J$. Radiat., 2011, 52: 103-109.

[53] J. Hong, Y. Zhong, Z. Yue, et al., Zincon-immobilized silica-coated magnetic $\mathrm{Fe}_{3} \mathrm{O}_{4}$ nanoparticles for solid-phase extraction and determination of trace lead in natural and drinking waters by graphite furnace atomic absorption spectrometry. Talanta, 2012, 94: 251- 256.

[54] K. Maryam, J.A. Tariq, K. Hossein, et al., The practical utility of the synthesis $\mathrm{FeNi}_{3}, \mathrm{SiO}_{2}, \mathrm{TiO}_{2}$ magnetic nanoparticles as an efficient photocatalyst for the humic acid degradation. Chemosphere, 2020, 239: 1-12.

[55] M.E.M. Pena, R.M. Mawalea, G.J.E. Conde, et al., Metal organic framework composite, nano- $\mathrm{Fe}_{3} \mathrm{O}_{4} @$ Fe-(benzene-1,3,5- tricarboxylic acid), for solid phase extraction of blood lipid regulators from water. Talanta. 2020, 207: 1-8.

[56] H. Nayeresadat, K. Faramarz, S.N.H. Mohammad, et al., Improving the antifungal activity of clove essential oil encapsulated by chitosan nanoparticles. Food Chem, 2019, 275: 113-122.

[57] M. Jérémie, P. Elisabetta, D.B. Ariane, et al., How to unravel the chemical structure and component localization of individual drug-loaded polymeric nanoparticles by using tapping AFM-IR. Analyst, 2018, 143: 5940-5949.

[58] K. Ondr, D. Tereza, K. Jakub, et al., Silica-coated manganite and Mn-based ferrite nanoparticles: a comparative study focused on cytotoxicity. J. Nanopart. Res., 2016, 100: 1-18.

[59] E. Hamideh, K. Mohammadreza, G. Keyvan, et al., Preparation and optimization of chitosan nanoparticles and magnetic chitosan nanoparticles as delivery systems using Box-Behnken statistical design. J. Pharm. Biomed. Anal., 2013, 80: 141-146.

[60] M. Piyasi, S. Kishor, C. Mousumi, et al., Oral insulin delivery by self-assembled chitosan nanoparticles: In vitro and in vivo studies in diabetic animal model. Mat. Sci. Eng., 2013, 33: 376-382.

[61] B.V. Nagavarma, N. Agavarma, S.Y. Hematk, et al., Formulation and evaluation of polymeric nanoparticulate gel for topical delivery. Int. J. Poly. Mat. Poly. Biomat., 2013, 63: 439-447.

[62] B. Rolando, G. Gabriele, F. Serena, et al., Biohydrogels with magnetic nanoparticles as crosslinker: Characteristics and potential use for controlled antitumor drug-delivery. Acta Biomat, 2012, 8: 4244-4252.

[63] R.S. Mohammad, M. Abel, and A.R. Mohammad, Synthesis and characterization of superparamagnetic nanoparticles coated with carboxymethyl starch (CMS) for magnetic resonance imaging technique. Carbohydrate Polymers, 2009, 78: 292-295.

[64] B. Alessandra, M. Federico, Chemometric methods for pectroscopy-based pharmaceutical analysis. Frontiers in Chemistry, 2018, 6(576): 1-14.

[65] H. Soheila, E. Pouneh, A.R. Hossein, et al., Optimization of preparation of chitosan-coated iron oxide nanoparticles for biomedical applications by chemometrics approaches. Internat. Nano Lett., 2013, 3(48): 1-5.

Copyright $₫$ Hesham Salem, Fatma Abo Elsoud, Ahmed Magdy, Dina Heshmat, Ahmed Abdelkareem Soliman, Kerolos Wissa, Kareem Hassan, and Rania Abdelrady. This is an openaccess article distributed under the terms of the Creative Commons Attribution License, which permits unrestricted use, distribution, and reproduction in any medium, provided the original author and source are credited. 